\title{
A randomized controlled trial to assess the effect of a ketamine infusion on tourniquet hypertension during general anaesthesia in patients undergoing upper and lower limb surgery.
}

\author{
Joyce Ongaya, Vitalis Mung'ayi, Thikra Sharif, Jimmie Kabugi
}

Department of Anaesthesia, Aga Khan University, East Africa

\begin{abstract}
Background: Tourniquet hypertension arising from tourniquet inflation remains a primary concern to the anaesthetist. One drug commonly used to manage tourniquet hypertension is ketamine. No studies have examined the effect of ketamine on tourniquet hypertension for a period of more than one hour or an infusion of the same.

Objective: To compare the effect of an intravenous infusion of ketamine versus placebo on tourniquet induced hypertension in patients undergoing upper and lower limb surgery under general anaesthesia.

Methods: Forty six adult patients scheduled for upper and lower limb surgery under general anaesthesia were randomized into two equal groups. The ketamine group received an intravenous bolus of $0.1 \mathrm{mg} / \mathrm{kg}$ of ketamine followed by an infusion of $2 \mathrm{ug} / \mathrm{kg} / \mathrm{min}$. The saline group received an intravenous bolus of physiological saline followed by an infusion of saline. All the patients were reviewed post-operatively. Data of the baseline characteristics, haemodynamic changes, post-tourniquet pain and side effects were collected. If post-tourniquet pain was present post-operatively, a visual analogue scale (VAS) was used to assess its severity.

Results: 46 patients successfully completed the trial. There were no significant differences between the groups for baseline patient demographics. The incidence of tourniquet hypertension was higher in the saline group (26.1\%) compared with ketamine group $(4.6 \%)$ with a $95 \%$ confidence interval. The difference was shown to be statistically significant ('P'<0.05). There was an increase in systolic blood pressure after 60 minutes of tourniquet inflation in the saline group but the difference was not statistically significant('P'>0.866). There were no significant differences between the groups as regards diastolic blood pressure and heart rate. VAS scores did not differ between the two groups. Statistically, there was no difference found between the two groups. Side effects were minimal in the ketamine group whilst in the saline group, nausea and vomiting were predominant but were also not statistically significant.
\end{abstract}

Conclusion: Based on the results of this study,there was a difference in the proportion of tourniquet hypertension between the ketamine and saline groups for patients undergoing upper and lower limb orthopaedic surgery under general anaesthesia.

Keywords: Randomized controlled trial, effect of a ketamine infusion, tourniquet hypertension, general anaesthesia, upper and lower limb surgery.

DOI: https://dx.doi.org/10.4314/ahs.v17i1.16

Cite as: Ongaya J, Mung'ayi V, Sharif T, Kabugi J. A randomized controlled trial to assess the effect of a Ketamine infusion on tourniquet bypertension during general anaesthesia in patients undergoing upper and lower limb surgery. Afri Health Sci. 2017;17(1): 122-132. bttps://dx.doi.org/10.4314/abs.v17i1.16

\section{Introduction}

The use of tourniquets dates back to early Roman times when various constricting bandages of different widths, were used to control haemorrhage during amputation ${ }^{1,2}$. Tourniquets are commonly used in orthopaedic surgeries
Corresponding author:
Vitalis Mung'ayi,
Department of Anaesthesia,
Aga Khan University, East Africa
Tel: +254203662157
Email: vitalis.mung'ayi@aku.edu, mungayi@gmail.com

to provide a bloodless operating field and good operating conditions. They have also been utilized in anaesthesia to prevent the central spread of local anaesthetic during intravenous regional anaesthesia also known as a Bier's block ${ }^{3}$.

The use of tourniquets has been associated with various changes ranging from minor to life threatening and even fatal in some cases. Systemic effects are usually related to inflation and deflation of the tourniquet, whereas local effects and complications may result from either direct pressure to the underlying tissues or ischemia in the tissues distal to the tourniquet ${ }^{2}$. The systemic effects include, increase in blood pressure and heart rate (tourni- 
quet hypertension or pain), temperature reduction in the non-perfused limb, production of anaerobic metabolites and hypercoagulability ${ }^{1,2}$. The local effects of tourniquet inflation include nerve, muscle, vascular and skin injury. After inflation for 30-60 minutes, patients may develop increases in heart rate systolic and diastolic arterial blood pressure that persist until tourniquet deflation, a phenomenon known as 'tourniquet pain or hypertension', Tourniquet pain is defined as a vague, dull pain in the limb associated with an increase in heart rate and blood pressure ${ }^{1,4}$. Prolonged tourniquet inflation is associated with a gradual increase in arterial blood pressure. The increase in blood pressure is resistant to analgesics and/ or an increase in the depth of anaesthesia ${ }^{5,6}$.

The mechanisms involved in tourniquet pain are not well understood but have been postulated to be conducted by A- delta and C- fibres which are thought to mediate somatic pain ${ }^{3,8-10}$.

The treatment is non-specific, and various methods have been used to counter the effects of tourniquet pain, these include intravenous bolus doses, local application, local anaesthesia, per oral administration, sub-cutaneous injection and regional anaesthesia, but most have been found to be unsatisfactory ${ }^{10,11,12}$.

Several studies have been carried out to investigate the most effective agent in attenuating tourniquet induced hypertension and ketamine has been found to be one of the most effective intravenous agent through its interaction with the $\mathrm{N}$-methyl D-aspartate receptor ${ }^{8,10,13}$.

Ketamine use for tourniquet pain/ hypertension has been widely investigated ${ }^{8,10,13,16}$. A study done by JW Park et al using $0.1 \mathrm{mg} / \mathrm{kg} \mathrm{I.V}$ and placebo on tourniquet induced hypertension during 60 minutes of tourniquet inflation concluded that low dose ketamine given pre-operatively prevented tourniquet hypertension and no patients were found to have ketamine induced psychomimetic effects during follow-up at 1 hour and 24 hours after waking. His recommendations for future studies included a longer monitoring period ${ }^{8}$.

Other studies have been done using $0.15 \mathrm{mg} \mathrm{kg}$ and $0.25 \mathrm{mg} / \mathrm{kg}$ and $1 \mathrm{mg} \mathrm{kg}$ of ketamine respectively ${ }^{13,16,17}$, however none of these studies have had intra-operative monitoring of blood pressure changes for more than 60 minutes, therefore no conclusions can be made on the effect of ketamine on tourniquet pain after 60 minutes of tourniquet inflation. No published literature exists on ketamine infusions for the management of tourniquet hypertension.

Based on these studies and the pharmacological profile of ketamine, our study was designed with a belief that an infusion of ketamine would attenuate tourniquet induced blood pressure changes and cater for analgesic requirements for procedures lasting longer than 60 minutes with tourniquet application.

The current study thus investigated the effect of a subanaesthetic infusion of ketamine on tourniquet induced blood pressure changes during general anaesthesia in patients undergoing orthopaedic surgery.

Our research question was: does an intravenous infusion of ketamine compared with placebo, significantly reduce the incidence of tourniquet induced hypertension in upper and lower limp surgery? Our null hypothesis was that there is no difference in blood pressure changes in patients receiving an intravenous infusion of ketamine compared with those receiving placebo after tourniquet inflation.

Our primary objective was to compare the effect of an intravenous infusion of ketamine versus placebo on the incidence of tourniquet induced hypertension in patients undergoing upper and lower limb surgery under general anaesthesia. Our secondary objectives were to assess heart rate changes associated with tourniquet use, assess efficacy of ketamine on post-operative tourniquet pain and determine the incidence of side effects associated with ketamine.

\section{Methods}

The study was performed following approval from the Ethical and scientific Research and Ethics Committee at the Aga Khan University, East Africa.

This was a prospective, randomized, controlled single blinded trial. The study was conducted between January and March 2013 at the Aga Khan University hospital, Nairobi. Since Nairobi is a cosmopolitan city, the patients served by the hospital cut across most racial groups present within the country. The study population included all adults of the age between 18 and 80 years undergoing elective upper and lower limb orthopaedic surgery. Patients were recruited from the pre-anaesthesia clinic, which runs on a daily basis, wards and the day-care unit. The sample population included adult American Society of Anaesthesiologists (ASA) physical status I and II pa- 
tients undergoing elective and emergency orthopaedic surgery with tourniquet application under general anaesthesia.

All ASA I and II patients aged 18-80 years scheduled for orthopaedic surgery were included in this study. Reasons for exclusion from the study were:

1. ASA physical status III and above

2. Pregnant women

3. Patients with hypertension, ischaemic heart disease and diabetes mellitus

4. Patients with dementia or a history of psychiatric illness

A sample size of 46 patients was sufficient to demonstrate a 30\% difference in the mean blood pressure variations between the two groups at 95\% confidence level and a power of $90 \%$. A study done by Tsuyoshi et al showed the percentage of patients who developed arterial pressure increase of more than $30 \%$ of base line values be- tween the ketamine group and the control group was 5\% and $40 \%$ respectively ${ }^{13}$. We therefore hypothesized that there would be a $30 \%$ less incidence of tourniquet hypertension in the ketamine group as compared with placebo. The sample size was calculated based on research done by Tsuyoshi et al. The reported mean systolic blood pressure in the ketamine group was $118.75 \mathrm{mmHg}(\mathrm{sd}=8.31)$ and for the control group was $126.13 \mathrm{mmHg}(\mathrm{sd}=6.1)$.

Using this information in the formula, assuming a $5 \%$ significance level and a power of $90 \%$, the required sample size was 23 patients in each group and a total of 46 patients.

The primary investigator approached prospective patients at the pre-anaesthetic clinic, ward and day-care unit. Patients were informed of the nature of the study, screened for eligibility and recruited if eligible. Eligible patients then received written and oral explanations on the purpose and nature of the study. A participant flow diagram is shown in figure 1.

Figure 1: Flow diagram of patient distribution

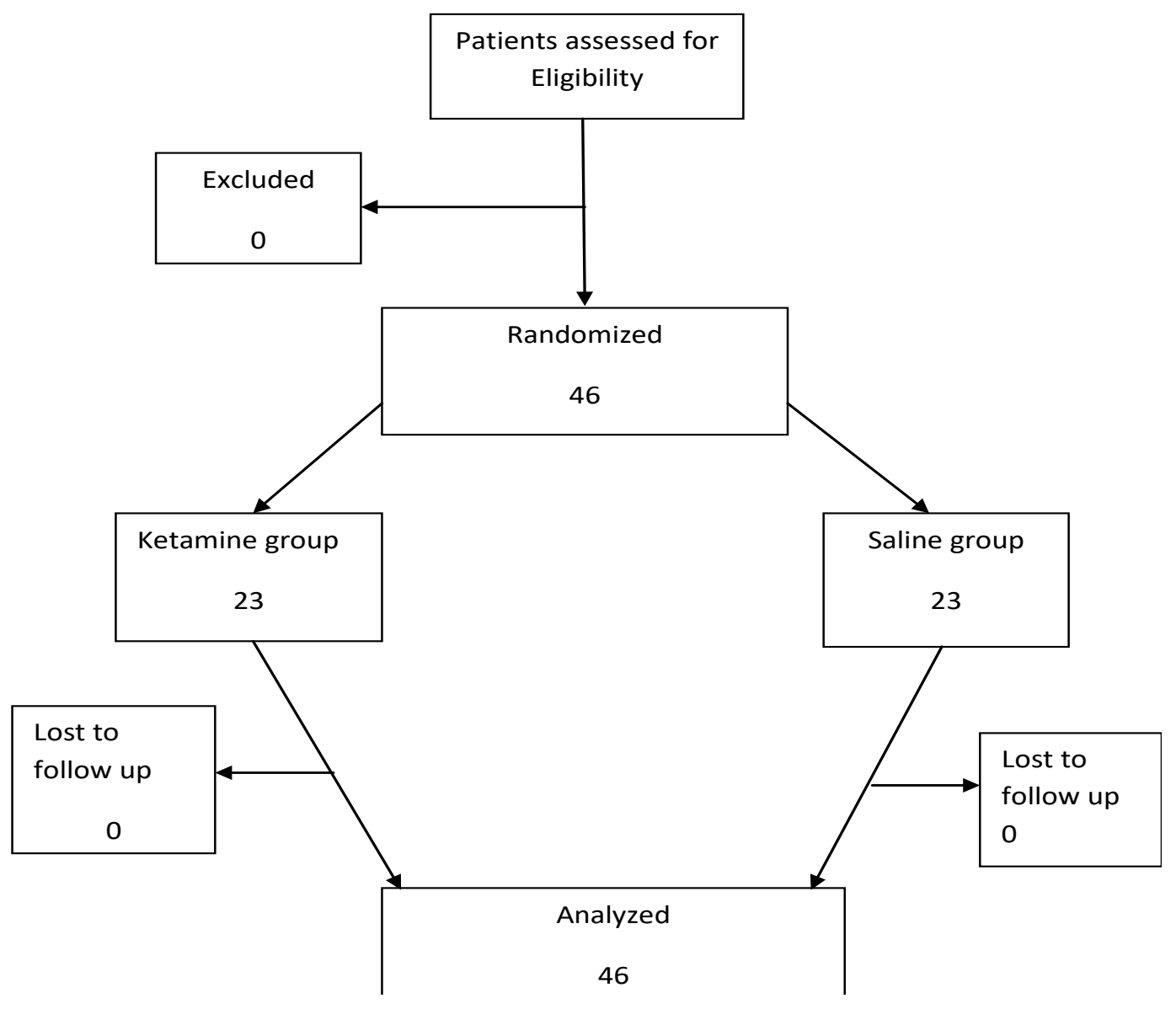


A randomization schedule was computer generated by a biostatistician (not otherwise involved in the study) and the code was provided to the primary investigator. The primary investigator then prepared the solution according to the randomization code, 15 minutes before the start of the procedure in the operating theatre. The solutions were labelled with the patients file number and initials of each solution to distinguish the two groups.

The randomization schedule specified the group to which each prospective patient was allocated upon enrolment in the trial.

Group 1: To receive I.V bolus of $0.1 \mathrm{mg} / \mathrm{kg}$ ketamine in a $10 \mathrm{ml}$ syringe followed by an infusion of $2 \mu \mathrm{g} / \mathrm{kg} / \mathrm{min}$ (ketamine group)

Group 2: To receive I.V bolus of physiological saline in a $10 \mathrm{ml}$ syringe followed by a continuous infusion of the same in a $20 \mathrm{ml}$ syringe (saline group)

All anaesthetists and anaesthetic assistants had prior sensitization and training as regards the meaning of the initials and management of adverse drug reactions. All patients, surgeons and other health care personnel involved in patient management were blinded to the study groups. On arrival to the operating theatre, standard monitoring was set up with automated non-invasive blood pressure measurement, electrocardiography and pulse oximetry. For the purpose of this study, tourniquet hypertension was defined as an increase of more than $30 \%$ in either systolic or diastolic blood pressure from baseline values. An I.V cannula was inserted and a three way tap attached. One port was used for the study solution and the other for induction of anaesthesia.

A standardized general anaesthetic protocol consisting of I.V midazolam $0.1-0.2 \mathrm{mg} / \mathrm{kg}$, I.V fentanyl $1 \mathrm{ug} / \mathrm{kg}$ and I.V propofol $2-2.5 \mathrm{mg} / \mathrm{kg}$ was used for induction of anaesthesia. The study drug and saline boluses were intravenously administered and infusions commenced respectively in both groups after induction of anaesthesia. I.V rocuronium $0.6 \mathrm{mg} / \mathrm{kg}$ was used to facilitate endotracheal intubation. Anaesthesia was maintained with $\mathrm{O}_{2} /$ AIR (1:1) in Isoflurane. Ventilation was controlled with a tidal volume of $8-10 \mathrm{mls} / \mathrm{kg}$ and a respiratory rate of $12-18 \mathrm{~b} / \mathrm{min}$ to maintain an end tidal carbondioxide concentration of 30-35. Anaesthesia was maintained with Isoflurane at $1-2 \%$. End tidal Isoflurane concentrations were adjusted to keep arterial pressures and heart rate, after induction and before tourniquet inflation within 20\% of their pre-induction values and thereafter maintained steady during the study period. Paralysis was maintained with rocuronium $0.15 \mathrm{mg} / \mathrm{kg}$ top up doses by decision of the anaesthetist and confirmed with a nerve stimulator.

Non-invasive arterial pressures, electrocardiography, pulse oximetry and capnography were monitored continuously. The concentration of end tidal Isoflurane was monitored by an anaesthetic gas monitoring system. At tourniquet deflation, all anaesthetics were terminated, the study drug and saline infusions in both groups stopped and the trachea extubated upon resumption of spontaneous respiration and after reversal of neuromuscular blockade with I.V $0.05 \mathrm{mg} / \mathrm{kg}$ neostigmine and I.V $0.02 \mathrm{mg} / \mathrm{kg}$ atropine. Patients received supplemental oxygen by mask and were transported to the post-anaesthetic care unit (PACU).

Patients received standard intra-operative analgesia prior to surgical incision consisting of i.v morphine $0.1 \mathrm{mg} / \mathrm{kg}$, i.v diclofenac $1 \mathrm{mg} / \mathrm{kg}$ and i.v paracetamol $15 \mathrm{mg} / \mathrm{kg}$. This continued for a period of 72 hours post-operatively. I.V. fentanyl $0.1 \mathrm{ug} / \mathrm{kg}$ was to be used as rescue analgesia if the patient's systolic blood pressure increased to $>40 \%$ of baseline in spite of end tidal Isoflurane concentrations of 1MAC. I.V. esomeprazole 40mg i.v was given to all patients as anti-ulcer prophylaxis and ondansetron $0.1 \mathrm{mg} /$ $\mathrm{kg}$ i.v was administered for opioid induced post-operative nausea and vomiting.

In the post-operative anaesthesia care unit, vital signs were recorded on arrival and a visual analogue scale score was taken one hour later.

Intra-operative and post-operative data was collected by the principal investigator or research assistant both in the operating theatre and PACU using the data collection form. The collected data was then entered by the principal investigator into an MS-Excel data base.

Data analysis was undertaken using the Analysis of variance, ANOVA statistical software and Fisher's exact test with the input of a statistician who was involved since the beginning of the proposal development. The same model was also used to assess the within group effect over time for blood pressure and heart rate. Student's t-test was used to analyze normally distributed variables and Chi square test for categorical data. Wilcoxon Rank-sum test was used to analyze skewed data. All data was presented as mean \pm standard deviation unless otherwise specified, and a 'P' $<0.05$ was considered to be statistically significant. 


\section{Results}

46 patients were enrolled into the study and were aged between 18-80 years, all undergoing elective lower and upper limb surgery. Their baseline characteristics are shown in table 1.

Table 1: Patients' baseline characteristics

\begin{tabular}{|l|l|l|l|}
\hline & Ketamine Group & Saline Group & \\
\hline & Mean (SD) & Mean (SD) & 'P' \\
\hline Age (years) & $49.4(19)$ & $44.8(15.5)$ & 0.376 \\
\hline Height (cm) & $158.8(9.4)$ & $162.5(10.8)$ & 0.219 \\
\hline Weight (Kg) & $76.7(10.7)$ & $78.9(13.3)$ & 0.547 \\
\hline Gender Male & $10(41.7)$ & $13(59.1)$ & 0.376 \\
\hline Gender Female & $13(58.3)$ & $10(40.9)$ & 0.376 \\
\hline & & & \\
\hline & Median(IQR) & Median(IQR) & \\
\hline ASA & $2(1,2)$ & $1.5(1,2)$ & 0.584 \\
\hline & & & \\
\hline
\end{tabular}

The two groups were similar with no statistical difference in their baseline characteristics. The mean age was 50 years in the ketamine group and 45 years in the saline group. The mean height was $158.8 \mathrm{~cm}$ in the ketamine group and $162.5 \mathrm{~cm}$ in the saline group. There were more females in the ketamine group as compared with the saline group but the difference was not statistically significant. Their ASA physical status I/II distribution was $11 / 12$ in the ketamine group and 11/12 in the saline group.

There were more lower-limb procedures than there were upper limb procedures ' $\mathrm{P}$ ' $>0.05$, therefore there was no statistical difference between the two groups as regards the type and site of the surgery (table 2 and 3 ).

Table 2: Surgery Site

\begin{tabular}{|l|l|l|l|l|}
\hline LIMB & SITE & $\begin{array}{l}\text { FREQ } \\
(\%) \text { KETAMINE } \\
\text { GROUP }\end{array}$ & $\begin{array}{l}\text { FREQ } \\
(\%) \text { SALINE } \\
\text { GROUP }\end{array}$ & $\begin{array}{l}\text { CREQ } \\
(\%)\end{array}$ \\
\hline Upper limb & ulna & $0(0)$ & $3(0)$ & $3(6.5)$ \\
\hline & radius & $1(4.2)$ & $2(9.1)$ & $3(6.5)$ \\
\hline & humerus & $1(4.2)$ & $0(0)$ & $1(2.2)$ \\
\hline & olecranon & $1(4.2)$ & $0(0)$ & $1(2.2)$ \\
\hline Lower limb & metacarpals & $0(0)$ & $2(9.1)$ & $2(4.3)$ \\
\hline & femur & $5(20.8)$ & $2(9.1)$ & $7(15.2)$ \\
\hline & knee & $7(29.2)$ & $6(9.1)$ & $13(28.3)$ \\
\hline & tibia & $7(29.2)$ & $5(22.7)$ & $12(26.1)$ \\
\hline & fibula & $1(4.2)$ & $2(9.1)$ & $3(6.5)$ \\
\hline & foot & $0(0)$ & $1(4.5)$ & $1(2.2)$ \\
\hline
\end{tabular}

Table 3: Type of Surgery

\begin{tabular}{|l|l|l|l|}
\hline TYPE OF SURGERY & $\begin{array}{l}\text { FREQ } \\
\text { (\%) } \\
\text { GROUP }\end{array}$ & $\begin{array}{l}\text { FREQ } \\
(\%) \quad \text { SALINE } \\
\text { GROUP }\end{array}$ & $\begin{array}{l}\text { CUM } \\
\text { FREQ } \\
(\%)\end{array}$ \\
\hline TKR & $5(25)$ & $4(13.6)$ & $9(19.6)$ \\
\hline ORIF & $15(62.5)$ & $13(59.1)$ & $28(60.9)$ \\
\hline Sequestrectomy & $2(8.3)$ & $1(4.5)$ & $3(6.5)$ \\
\hline Reconstruction & $0(0)$ & $1(4.5)$ & $1(2.2)$ \\
\hline Arthroscopy & $1(4.2)$ & $3(13.6)$ & $4(8.7)$ \\
\hline Others & $0(0)$ & $1(4.5)$ & $1(2.2)$ \\
\hline & & & \\
\hline
\end{tabular}

Abbreviations: Freq- Frequency. Fisher's exact test 0.543 
A greater proportion of patients in the saline group developed tourniquet induced hypertension compared with the ketamine group (26.08\% versus $4.35 \%)$ and this was statistically significant $(\mathrm{P}=0.02)$ as seen in figure 2 .

\section{Figure 2: Incidence of tourniquet induced hypertension}

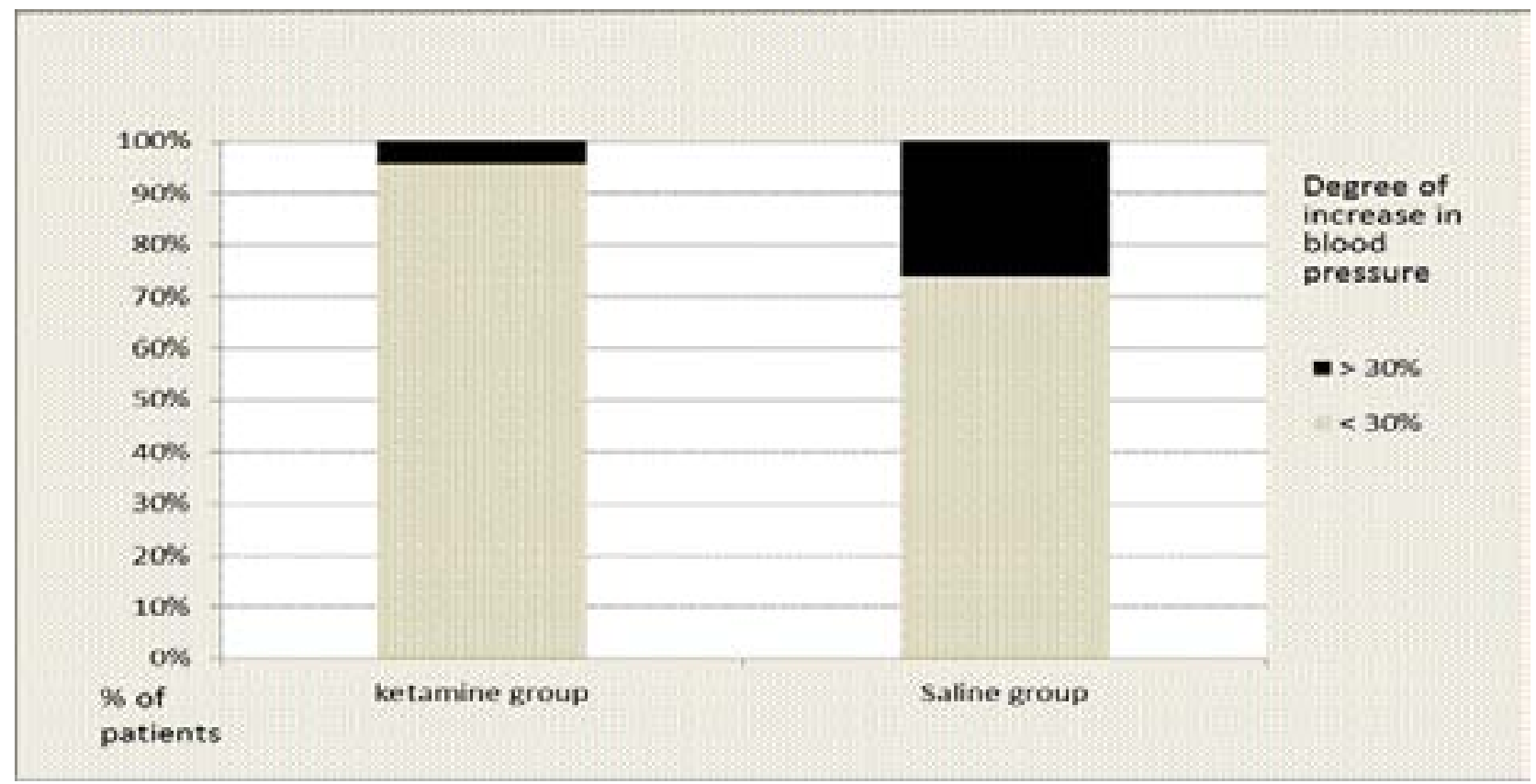

There was an increase in mean systolic blood pressure after 60 minutes of tourniquet application in the saline group but not in the ketamine group (figure 3). The be- tween groups test indicates that the variable group is not significant i.e. ${ }^{~} \mathrm{P}=0.866$. The groups do not change in systolic blood pressure over time. The difference is therefore not statistically significant.

Figure 3: Change in mean systolic blood pressure over time

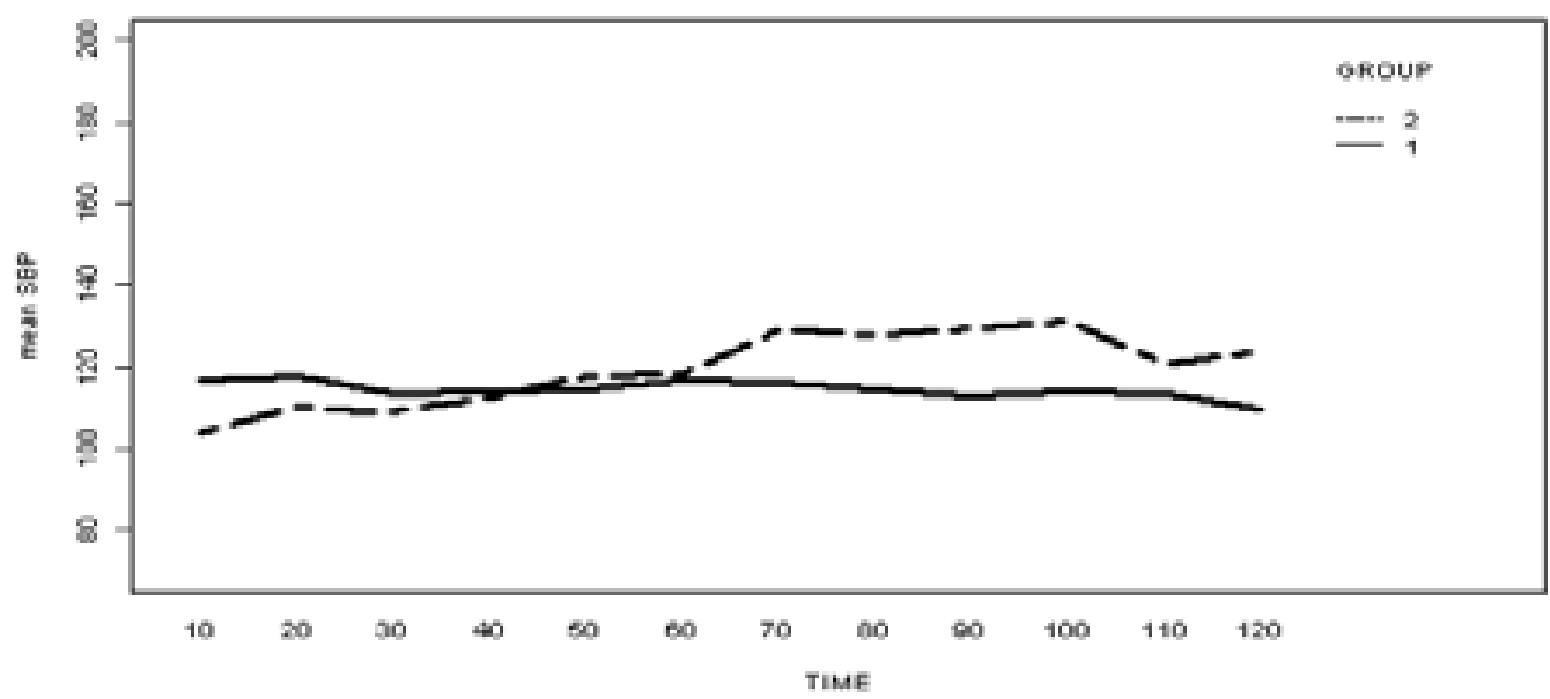

Key: Group 1- ketamine group, group 2- saline group Abbreviations: SBP- Systolic blood pressure

There was no difference in mean diastolic blood pressure

between both groups after 60 minutes of tourniquet application (figure 4). 
Figure 4: Change in mean diastolic blood pressure over time

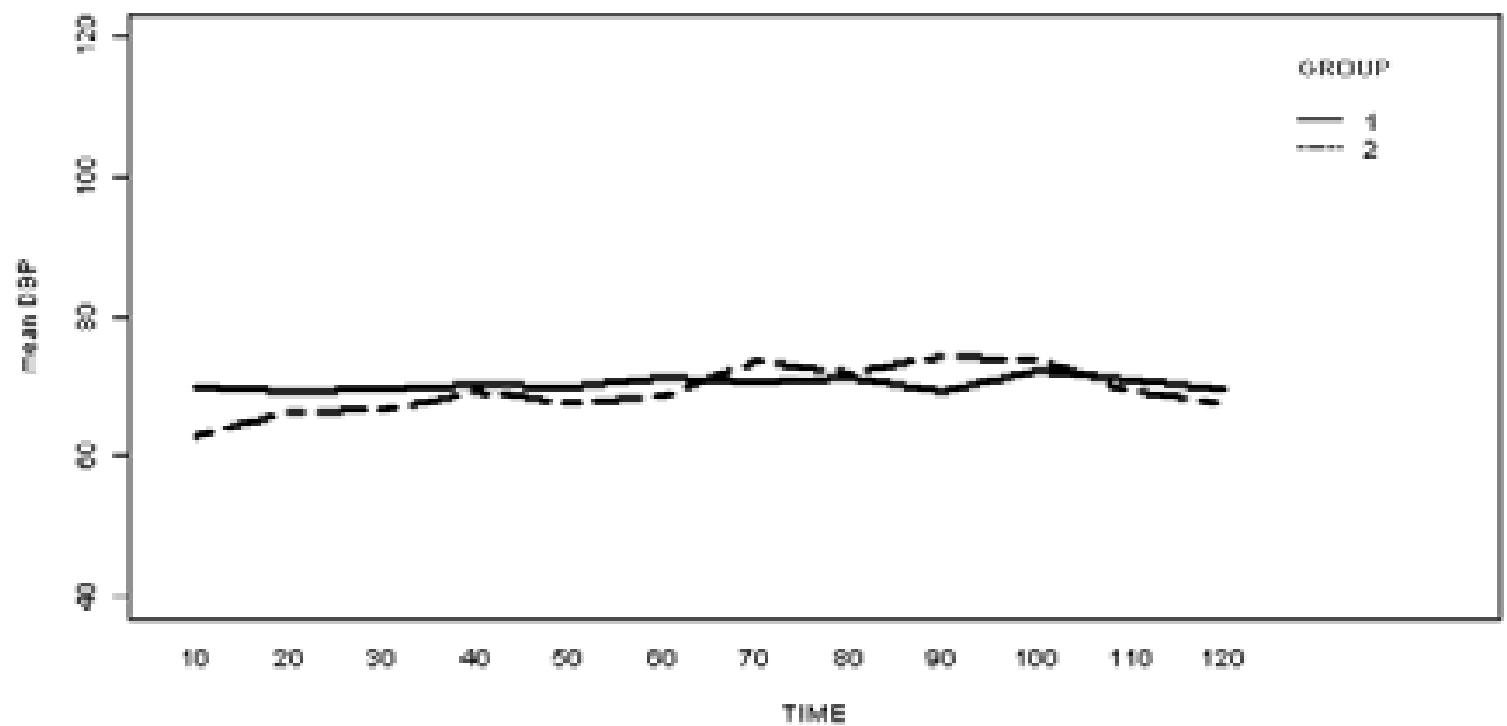

In the ketamine group there was no significant increase in rate after 60 minutes of tourniquet inflation (figure 5). mean heart rate 60 minutes after tourniquet inflation but The between groups test indicates that the variable group in the saline group there was an increase in mean heart is not significant i.e. $\mathrm{P}=0.8449$.

Figure 5: Change in mean heart rate over time

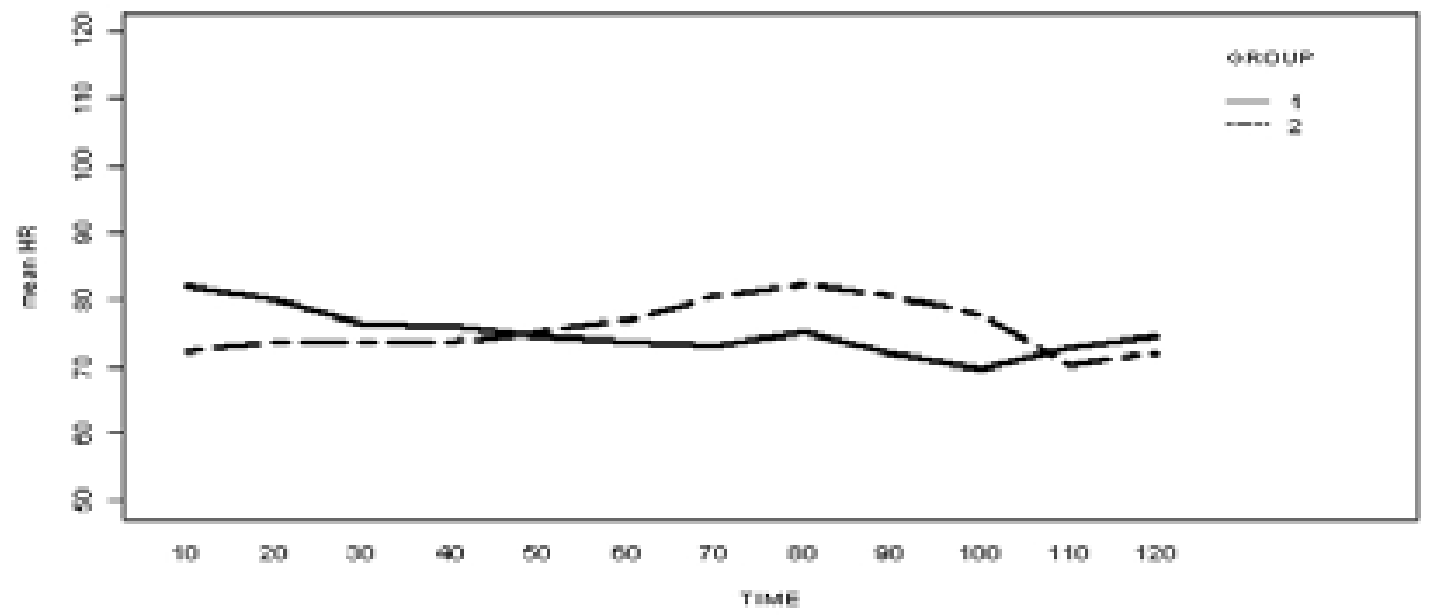

Key: Group 1- ketamine group, group 2- saline group Abbreviations: HR - Heart rate

There was no difference in mean end tidal isoflurane after 60 minutes of tourniquet inflation in both groups (figure 6). The between groups test indicates that the variable group is not significant i.e. 'P' $=0.853$. The within subject test indicate that there is not a significant time effect i.e. $\mathrm{P}=0.693$, meaning that the groups do not change in SBP over time. No patient experienced significant hypertension (arterial blood pressure $>40 \%$ of baseline) during the observation intra-operatively. 
Figure 6: Change in mean end tidal isoflurane over time

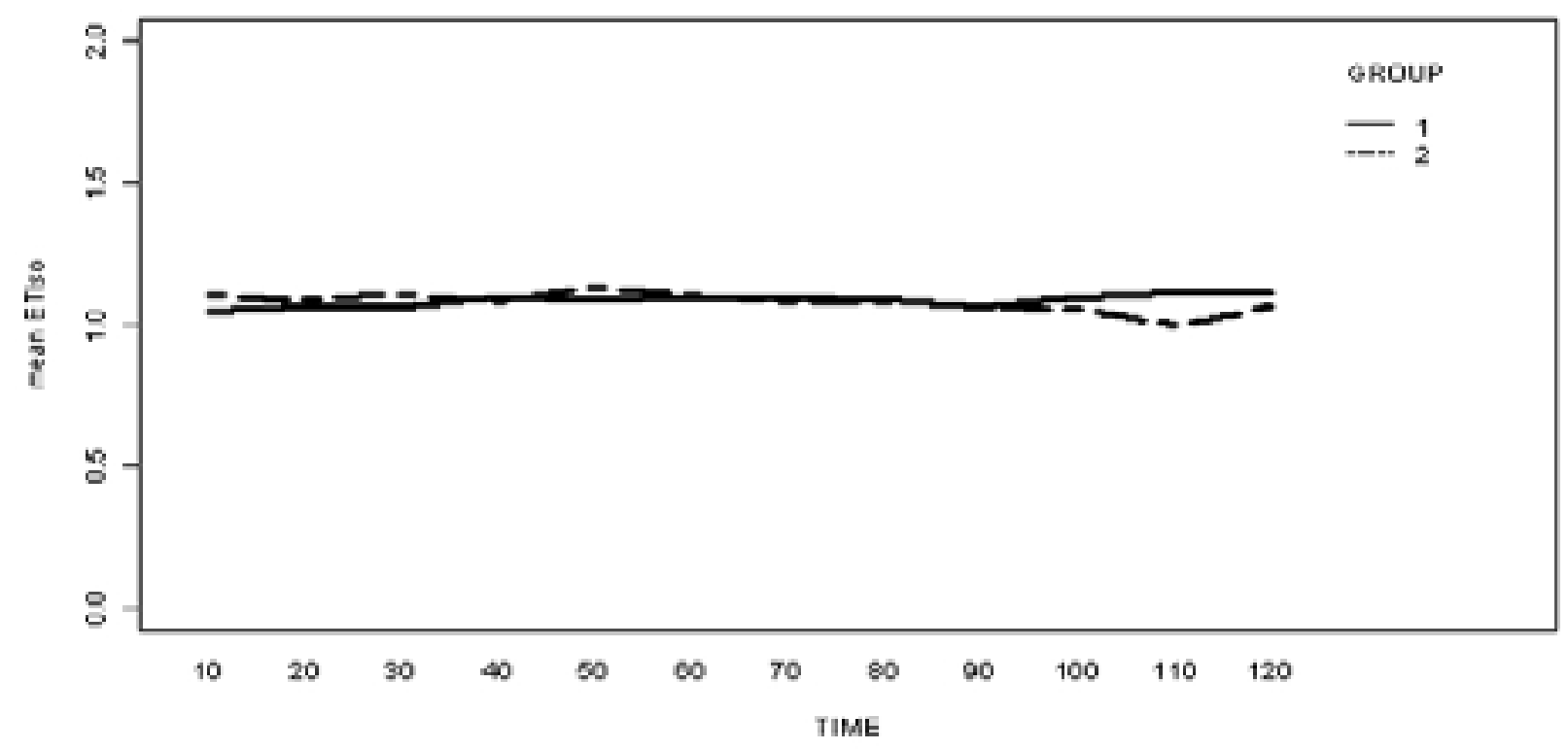

The Kaplan Meier curve (figure 7) demonstrates that the time to onset of hypertension was similar in both groups

and that there was no difference in the proportion of hypertension after 60 minutes of tourniquet time in both groups.

Figure 7: Kaplan Meier survival time to hypertension

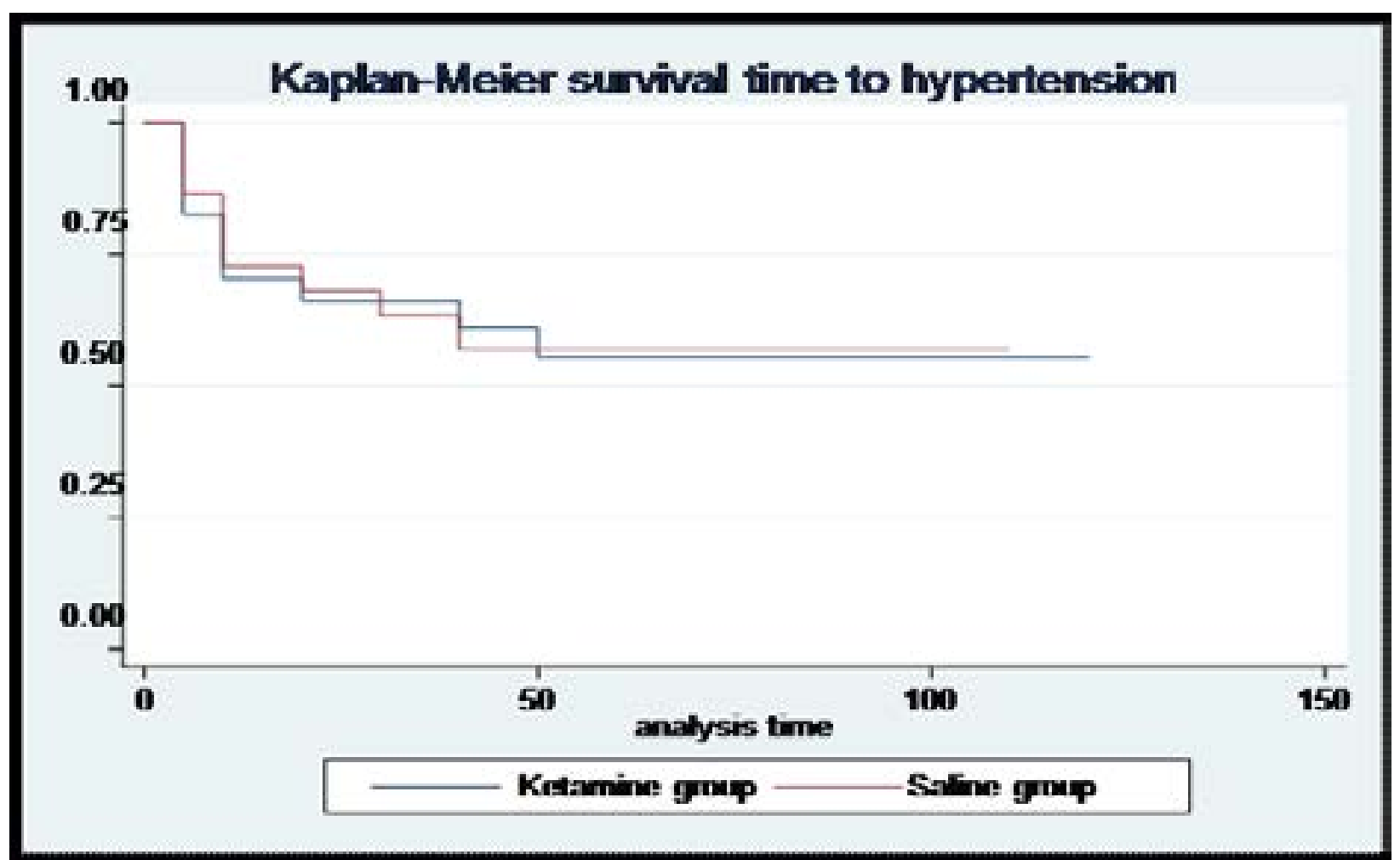


The Wilcoxon rank-sum test was used to compare VAS scores between the two groups (table 4) and it showed that there was no difference between the two groups as regards VAS scores $(\mathrm{P}=0.546)$.

\section{Table 4: VAS Scores}

\begin{tabular}{|l|l|l|}
\hline & Ketamine group & Saline group \\
\hline & Median (IQR) & Median (IQR) \\
\hline VAS & $2(1,3)$ & $2(1,5)$ \\
\hline & & \\
\hline
\end{tabular}

Abbreviations: VAS- visual analogue scale. Wilcoxon rank-sum test

The fisher's exact test was used to compare frequency and differences in proportion of side effects between the two groups (table 5 and 6). The difference was not statis- tically significant, $\mathrm{P}=0.825$. No patient complained about ketamine related psychotomimetic effects or psychological disturbances on follow-up in the post-anaesthesia care unit (PACU).

\section{Table 5: Side effects}

\begin{tabular}{|l|l|l|l|}
\hline & Side Effects & Ketamine group & Saline group \\
\hline & & Freq $(\%)$ & Freq $(\%)$ \\
\hline & Yes & $3(12.5)$ & $3(13.6)$ \\
\hline & No & $20(87.5)$ & $20(86.4)$ \\
\hline & & & \\
\hline
\end{tabular}

Fisher's exact test

\begin{tabular}{|l|l|l|}
\hline Type of S.E & Ketamine group & Saline group \\
\hline & Freq $(\%)$ & Freq (\%) \\
\hline Nausea/vomiting & $2(8.3)$ & $3(13.6)$ \\
\hline Headache & $1(4.2)$ & $0(0)$ \\
\hline Blurred vision & $0(0)$ & $0(0)$ \\
\hline Painful micturition & $0(0)$ & $0(0)$ \\
\hline Psychotomimetic effects & $0(0)$ & $0(0)$ \\
\hline & & \\
\hline
\end{tabular}

Fisher's exact test

\section{Discussion}

Tourniquet hypertension was defined as an increase of more than $30 \%$ in either systolic or diastolic blood pressure, from baseline values. It was found that the incidence of tourniquet induced blood pressure changes was $4.35 \%$ in the ketamine group and $26.08 \%$ in the saline group (figure 2), which is almost similar to previous studies which showed the incidence to be $28.6 \%$ in the saline group and $7.1 \%$ in the ketamine group, thus rejecting the null hypothesis at the beginning of the study ${ }^{8}$.

The results of this study showed that an intra-operative small bolus dose of I.V ketamine followed by an infusion of low dose ketamine, prevented systolic blood pressure increases and heart rate increase in the ketamine group compared with the saline group after 60 minutes of tourniquet inflation in patients under general anaesthesia. The difference after analysis was not found to be statistically significant for systolic blood pressure and heart rate (Figure 3 and $5, \mathrm{P}=0.866$ and $\mathrm{P}=0.8449$, respectively). The same was found for diastolic blood pressure $\mathrm{P}=0.578$.

End tidal isoflurane concentrations did not change much over time from tourniquet inflation to deflation and the difference was not found to be statistically significant between the two groups $(\mathrm{P}=0.853)$. This result was similar 
to previous studies ${ }^{8,13}$, although both studies used different inhalational agents, namely enflurane and sevoflurane. Both agents do not differ much from isoflurane in terms of haemodynamics although enflurane has the potential to cause arrhythmias as it sensitizes the myocardium to catecholamines.

VAS scores for post tourniquet pain did not vary much between both groups although higher scores were reported in the saline group. Fisher's exact test showed the $\mathrm{P}>0.05$ which means that the difference was not statistically significant. Previous studies did not assess post tourniquet pain although they reported lower pain scores at the surgical site 24 hours after the surgery in the ketamine group as compared with the control group ${ }^{13}$.

A recent Cochrane review analyzed several studies with regard to the effectiveness of ketamine administered peri-operatively for acute post-operative pain, the authors concluded that low dose ketamine was effective in decreasing morphine requirements in the first 24 hours after surgery and decreases the incidence of post-operative nausea and vomiting, and adverse effects were mild or absent ${ }^{14}$. This offers an explanation as to the reason why the saline group had a higher incidence of nausea and vomiting. None of the patients in the ketamine group was found to have psychotomimetic reactions which are the most serious side effects of ketamine probably due to the sub-anaesthetic doses used in this study.

The study was a single blinded randomized controlled trial that was adequately powered to detect the primary outcome. The calculation of the power in the current study was based on other randomized controlled trials. The incidence of tourniquet hypertension was found to be higher in the saline group than in the ketamine group concurring with other studies. This is the first study to use ketamine as an infusion for tourniquet induced hypertension. All other studies used ketamine as an intravenous bolus before tourniquet application.

\section{Limitations}

Our study has several limitations. Firstly, it was not double blinded or multi-centred. Secondly the study was performed on patients under general anaesthesia, therefore the effect of ketamine alone, on tourniquet induced hypertension and the causal relationship between tourniquet induced hypertension and tourniquet induced pain could not be evaluated. Thirdly, surgical procedures were not standardized therefore discrepancies in the response to tourniquet application could have contributed to finding no differences between the two groups. Lastly, the depth of anaesthesia may not have been identical among the patients because Bispectral index (BIS) or Electroencephalogram (EEG) monitoring was not used to ensure similar anaesthetic depth.

\section{Conclusion}

On the basis of the results of this study, there was a significant difference in the two groups when they were randomly assigned to a control and intervention group, as regards the incidence of tourniquet hypertension. Although JW Park, H Valli and Tsuyoshi ${ }^{7,8,13}$ also showed a difference, their study differed from ours in some important ways, namely, none of their studies used an infusion of ketamine after the initial bolus and we know from the pharmacology of ketamine the duration of analgesia is 40 minutes if given as an intravenous bolus dose $\mathrm{e}^{15}$ and the studies quoted had tourniquet application times of 60 minutes, while in our study tourniquet time was more than 60 minutes. Consequently we believe that our study mirrors clinical practice more closely and is thus applicable to more clinical scenarios.

\section{Conflict of interest}

\section{References}

1. Noordin S, McEwen J, Kragh JF, Eisen A, Masri B. Surgical tourniquets in orthopaedics. The Journal of Bone and Joint Surgery. American volume. 2009 Dec;91(12):2958-67.

2. Kam PC, Kavanagh R, Yoong FF, Kavanaugh R. The arterial tourniquet: pathophysiological consequences and anaesthetic implications. Anaesthesia. 2001 Jun; 56(6):53445.

3. Estebe JP, Le Naoures, Chemaly L, Ecoffey C. Tourniquet pain in a volunteer study: effect of changes in cuff width and pressure. Anaesthesia. 2000 Jan;55(1):21-6.

4. KaufmanRD, Walts LF. Tourniquet-induced hypertension. British Journal of Anaesthesia. 1982;54(3):333-6.

5. Townsend HS, Goodman SB, Schurman DJ, Hackel A, Brock-Utne JG. Tourniquet release: systemic and metabolic effects. Acta Anaesthesiologica Scandinavica. 1996;40(10):1234-7. nnn

6. Takada M, Fukusaki M, Terao Y, Kanaide M, Yamashita K, Matsumoto S, et al. Preadministration of low-dose ketamine reduces tourniquet pain in healthy volunteers. Journal of Anesthesia. 2005;19(2):180-2. 
7. Valli H, Rosenberg PH. Effects of three anaesthesia methods on haemodynamic responses connected with the use of thigh tourniquet in orthopaedic patients. Acta Anaesthesiologica Scandinavica. 1985 Jan;29(1):142-7.

8. Park J-W, Jung Y-H, Baek C-W, Kang H, Cha S-M. Effects of low dose ketamine on tourniquet-induced haemodynamic responses during general anaesthesia. The Journal of International Medical Research. 2007;35(5):600-8. 9. Hodgson AJ. A proposed etiology for tourniquet-induced neuropathies. Journal of Biomechanical Engineering. 1994;116(2):224-7.

10. Gorgias NK, Maidatsi PG, Kyriakidis M, Karakoulas $\mathrm{K}$, Alvanos DN, Giala MM. Clonidine versus ketamine to prevent tourniquet pain during intravenous regional anesthesia with lidocaine. Regional Anesthesia and Pain Medicine. 2001;26(6):512-7.

11. Tsai YC, Lai YY, Chang CL. Comparison of the effect of EMLA cream, subcutaneous ring anaesthesia and a double cuff technique in the prevention of tourniquet pain. British Journal of Anaesthesia. 1993 Apr;70(4);394-6. 12. Warncke T, Jørum E, Stubhaug A. Local treatment with the N-methyl-D-aspartate receptor antagonist ketamine, inhibit development of secondary hyperalgesia in man by a peripheral action. Neuroscience Letters. 1997 May 9;227(1):1-4.

13. Satsumae T, Yamaguchi H, Sakaguchi M, Yasunaga T, Yamashita S, Yamamoto S, et al. Preoperative small-dose ketamine prevented tourniquet-induced arterial pressure increase in orthopedic patients under general anesthesia. Anesthesia and Analgesia. 2001 May;92(5):1286-9.

14. Rf B, Jb D, Ra M, Ea K. Perioperative ketamine for acute postoperative pain ( Review ). 2010;(11).

15. DubyJJ. Ketamine : Pharmacology, Controversy and Role. 2010;10(2):2-3.

16. Menigaux C, Guignard B, Fletcher D, Sessler DI, Dupont X, Chauvin M. Intraoperative small-dose ketamine enhances analgesia after outpatient knee arthroscopy. Anesthesia and analgesia. 2001 Sep;93(3):606-12.

17. Lee DH, Lee DL, Kim Sy, Kim JM, Lee HM. Magnesium sulphate attenuates tourniquet induced hypertension and spinal C-fos mRNA expression; a comparison with ketamine. J Int Med Res 2006; 34(6) 573-84. PubMed 